\title{
草原生态系统损害鉴定评估体系及管理建议
}

\author{
李 爽 ${ }^{1,2}$,马一丁 ${ }^{1,2, *}$, 付 晓 ${ }^{1}$, 严 岩 ${ }^{1,2}$ \\ 1 中国科学院生态环境研究中心,城市与区域生态国家重点实验室, 北京 100085 \\ 2 中国科学院大学, 北京 100049
}

\begin{abstract}
摘要:如何科学合理的确定生态系统是否受到损害、损害到什么程度、损害赔偿如何估算等问题已成为环境管理、环境司法工作 所面临的紧迫的现实问题。以生态学、复合生态系统生态学、可持续发展的理论为指导,基于损害基线判定损害事件发生,借此 鉴定草原生态系统损害状态及鉴定等级,并从受损生态系统的功能与结构角度提出草原生态环境损害鉴定评估体系,开展对草 原生态系统损害的程度评估; 最后针对草原生态系统的损害赔偿价值提出量化评估的方法,借此推动生态环境损害鉴定评估法 的制定和完善生态环境损害鉴定评估技术体系两个方面提出管理建议。
\end{abstract}

关键词:生态系统损害;评估、鉴定、赔偿、管理体系

\section{The system for identification, assessment and compensation value quantification of grassland ecosystem damage and management suggestions}

\author{
LI Shuang $^{1,2}$, MA Yiding ${ }^{1,2, *}$, FU Xiao $^{1}$, YAN Yan $^{1}$ \\ 1 State Key Laboratory of Urban and Regional Ecology, Research Center for Eco-Environment Sciences, Chinese Academy of Sciences, Beijing 100085, China \\ 2 University of Chinese Academy of Sciences, Beijing 100049, China
}

\begin{abstract}
With the rapid population growth, economic and social development, ecosystem damage incidents occur frequently, which has a negative effect on the structure and function of different ecosystems. The quantitative assessment of eco-environmental damage and the improvement of the technical system of identification and assessment are the basis of a scientific and reasonable eco-environmental damage compensation system. Currently, there is no special regulation on ecoenvironmental damage identification and assessment in China, so it is unable to provide systematically legal support for the eco-environmental damage identification and assessment. At the same time, due to the imperfection of the eco-environmental damage identification and assessment technical system, it is hard to conduct related work. The issues of how to scientifically and reasonably determine whether the ecosystem has been damaged, to what extent it has been damaged, and how to estimate the compensation value have become urgently practical problems faced by environmental management and environmental justice. Grassland is the largest terrestrial ecosystem in China, which plays important roles in conserving water, fixing carbon and releasing oxygen, regulating climate and maintaining species diversity and so on. A systematic study of identification and assessment of the grassland ecosystem damage is of great significance to China's ecological security and construction of ecological civilization. Guided by the theories of ecology, complex ecosystem ecology and sustainable development, this paper builds up a system of identifying the damage incident based on damage baseline and identifies the seriousness and status of grassland ecosystem damage. The assessment system of grassland ecosystem damage is
\end{abstract}

基金项目: 国家重点研发计划课题(2016YFC0503603)

收稿日期: 2020-03-10; 采用日期: 2021-06-02

*通讯作者 Corresponding author.E-mail: ydma@ rcees.ac.cn 
then put forward from the perspective of the function and the structure of the damaged ecosystem. Finally, the method of compensation value evaluation and quantification for grassland ecosystem damage is proposed. By doing so, the establishment of regulations for identification and assessment of eco-environment damage can be promoted, and the management suggestions can be proposed for the technical system of ecosystem damage identification and assessment. The construction of the system for identification, assessment and compensation value quantification of grassland ecosystem damage is a useful attempt for grassland ecosystem management.

Key Words : ecosystem damage; assessment identification compensation management system

随着人口的快速增长和经济社会的快速发展,生态系统损害事件频发, 导致不同生态系统的结构和功能 受到不同程度的影响与破坏 ${ }^{[1-3]}$ 。据统计, 我国生态环境损害赔偿事件以每年 $25 \%$ 比例上 升 $^{[4]}$,使得业界对 于生态环境损害的鉴定评估以及赔偿价值量化的手段需求十分迫切 ${ }^{[3]}$ 。自 2011 年起, 原环保部陆续发布一 系列针对生态环境损害评估鉴定的相关指导性文件, 就生态环境损害鉴定评估工作的原则、内容、技术方法等 做出较为系统的规定, 如 2015 年 9 月和 12 月, 《生态文明体制改革总体方案》和《生态环境损害赔偿制度改 革试点方案》先后出台, 提出了生态环境损害赔偿制度改革的总体要求与目标; 2016 年, 《关于规范环境损害 司法鉴定管理工作的通知》和《生态环境损害鉴定评估技术指南总纲》先后颁布,对生态环境损害鉴定评估的 一般性原则、程序、内容和方法进行了规定。上述文件,使得我国生态环境损害鉴定评估工作得到了细化和有 针对性的开展 ${ }^{[5-9]}$ 。

生态环境损害的定量化评估以及鉴定评估技术体系的完善,是科学合理的生态环境损害赔偿制度的基 础 ${ }^{[10]}$ 。目前, 国内外关于生态环境损害评估与管理的研究已经取得了一些成果 ${ }^{[11-18]}$ 。Peter 等 ${ }^{[19]}$ 建议从理 解森林自然扰动生态学过程的基础上对森林资源进行管理; Lawrence 等 ${ }^{[20]}$ 对自然资源环境损害评估中量化 自然资源损害和生态服务减少、因果关系评估和基线建立三部分进行探讨。然而, 我国环境损害评估研究起 步较晚, 针对森林、草原、淡水、农田等生态系统的损害鉴定、评估与量化赔偿等技术方法的研究仍较为缺失, 需要不断细化与深人的研究。

草原是我国面积最大的陆地生态系统,约占国土面积的 $42 \%{ }^{[21]}$, 具有涵养水源、固碳释氧、调节气候、维 持物种多样性、保障畜牧业发展等重要作用, 因此就草原生态系统损害状态及等级鉴定进行系统研究,对于国 家生态安全及生态文明建设都具有重要意义 ${ }^{[22-24]}$ 。然而, 人为活动的过度干扰, 对草原生境造成了极大地破 坏。目前, 由于草原生态系统损害鉴定评估工作存在着责任分散、管理制度不健全等问题,造成了草原生态环 境损害司法实践过程中 “告状难、受理难、审判难、执行难”及行政处罚缺乏量化依据的困境。纠其根本乃是 现有法律法规对生态环境损害鉴定及损害赔偿评估工作无法提供系统支撑。因此, 研究如何科学、系统地对 生态系统损害程度进行度量和鉴定、采用何种技术措施评估及损害赔偿范围的确定等问题,对于保障经济与 生态环境协调可持续发展具有重要意义。

本文以生态学、复合生态系统生态学、可持续发展的理论为指导,基于损害基线判定损害事件发生,借此 鉴定草原生态系统损害状态及鉴定等级; 并从受损生态系统的功能与结构等角度提出草原生态环境损害鉴定 评估体系, 开展对草原生态系统损害的程度评估; 最后针对草原生态系统的损害赔偿价值提出量化评估的方 法。本研究旨在, 从推动生态环境损害鉴定评估法的制定和完善生态环境损害鉴定评估技术体系两个方面提 出管理建议。

\section{1 草原生态系统是否发生损害及损害等级鉴定}

草原生态系统损害指因自然或人为因素导致的环境要素和生态系统结构发生不利改变, 及生态系统功能 的退化或丧失。损害与否的判定标准, 首先需确定生态损害基线水平 ${ }^{[5]}$ 。损害基线可依据如下确定:

(1) 利用评估区域的历史数据来确定本区域生环境污染现象或破坏生态行为发生。数据来源可基于常 
规检测、专项调查、统计报表、学术研究等数据的收集, 反映人群健康、财产状况和生态环境状况的历史数据;

(2)利用与评估区域相类似、未经污染或破坏的类似现场数据,即形成“对照区域”。“对照区域”的选择 强调与评估区域的人群特征、生态系统功能和生态服务水平的特征具有可比性或高度相似性,通过参照“对 照区域”的状态确定评估区域的基线;

(3) 通过构建污染物浓度与人体健康指标、财产损害程度、生物量或生境丰度等损害评价指标间的计量一 反应关系模型来确定基线。

在确定损害基线的基础上,鉴定评估区域是否发生生态损害现象,可根据以下具体情形来断定:

(1) 评估区域内环境介质中污染物浓度超过基线水平或国家及地方的环境质量标准,且造成的影响及破 坏短期内无法恢复。

(2)评估区域发生环境污染或生态破坏行为后, 与基线状态相比,关键指示物种的数量或物种种群密度 (生物量) 的差异具有显著性。

(3) 评估区域发生环境污染或生态破坏行为后, 与基线状态相比,动植物物种种群组成、生物多样性等的 差异具有显著性。

(4)评估区域发生环境污染或生态破坏行为后,与基线状态相比,生物体外畸形,骨骼变形或内部器官、 软组织畸形,组织病理学水平的损害等发生率的差异具有显著性。

在确定评估区域生态系统损害确实发生的基础上,本研究根据受损生态系统是否可恢复至生态系统基线 为标准将生态系统损害分为四个等级,如表 1 所示。

\section{表 1 生态系统损害等级}

Table 1 The level of ecosystem damage identification and assessment

\begin{tabular}{ll}
\hline 损害程度 & 判定指标 \\
Extent of damage & Judgment index \\
\hline 轻微损害 & 1. 生态系统结构没有改变 \\
Minor damage & 2. 生态系统服务能力有所降低,量化后降低小于 $10 \%$ \\
& 3. 停止人为干扰后可自然恢复至生态系统基线水平 \\
轻度损害 & 1. 生态系统结构轻度改变,但主要建群种没有改变 \\
Mild damage & 2. 生态系统服务能力降低,量化后降低 $20 \%$ - $50 \%$ \\
& 3. 需借助人工修复手段恢复至生态系统基线状态 \\
中度损害 & 1. 生态系统结构改变,主要建群种改变 \\
Moderate damage & 2. 生态系统服务能力显著降低或丧失,量化后降低 $50 \%$ 一 $70 \%$ \\
& 3. 人工修复手段不能恢复至生态系统基线,但可以通过景感营造重建满足人类需求的生态系统 \\
重度损害 & 1. 生态系统结构改变,主要建群种丧失 \\
Severe damage & 2. 生态系统服务功能丧失,量化后降低大于 $70 \%$ \\
& 3. 通过各种途径均无法恢复 \\
\hline
\end{tabular}

轻微损害是指生态系统结构没改变,但生态系统服务能力有所降低,损害行为在生态系统承载能力范围 内, 当损害行为停止后,生态系统无需人为介人,可在较短时间内自行恢复至基线水平,例如草原中的野火、小 范围的森林火灾等。

轻度损害是指损害行为导致生态系统结构发生改变,生态系统服务能力下降,但主要建群种没有改变,仅 依靠自然恢复,无法使生态系统恢复至基线水平,需要人为干预,采取相应的生态修复措施,在修复措施实施 后, 通过自然恢复及人工后期维护,生态系统在短时间内恢复至基线水平,例如人工林砍伐,一定强度的草原 放牧。

中度损害是指损害行为导致生态系统结构发生改变,生态系统服务能力显著降低,无法依靠自然恢复达 到生态环境基线水平, 即使通过人为干预,无法恢复至生态系统的基线水平。但可以通过景感营造重建满足 人类需求的生态系统,例如草原露天矿边坡重塑。 
重度损害是指损害行为致使生态系统结构发生重大改变,生态系统服务完全丧失,已无法通过任何恢复 手段进行恢复。此种损害是一种不可逆的永久性损害。也是最为严重的损害程度,例如核污染及核泄漏, 火 山爆发等。人类的生存与发展离不开对自然资源的利用,但应在获取自身利用的同时,保证其行为在生态系 统可承受的范围内,避免对生态系统造成不可逆损害。

\section{2 草原生态系统损害鉴定评估体系}

良好的生态系统服务能力需以具备完整、稳定、连续及可持续特征的健康的生态系统为基础。因此,健康 的生态系统, 是保障生态文明建设和可持续发展的重要基础 ${ }^{[25]}$ 。生态系统损害鉴定评估的根本目标也是要 将受损生态系统恢复至基线状态或者相对健康状态。损害鉴定评估工作则是实现这一目标的重要途径。因 此在确定损害鉴定评估体系时,其所选取的指标应能全面反映使受损生态系统恢复至基线或者健康生态系统 的各项特征。

对于草原生态系统损害鉴定工作,应建立一套完整和科学的评估逻辑体系,如图 1 所示。首先,草原生态 系统损害鉴定评估工作需选取与受损生态系统立地条件相同或相近的未受人为干扰的生态系统作为基线水 平,调查和测试其度量基准的各项指标为基准指标, 度量基准主要从草原生态系统结构和功能两方面考量。 其次, 就受损生态系统的损害程度进行评估, 主要从受损生态系统结构和功能两方面进行测度, 确定生态系统 的损害程度。其中草原生态系统结构指标主要包括: 群落组成、物种多样性、建群种状态、植被覆盖度、生物

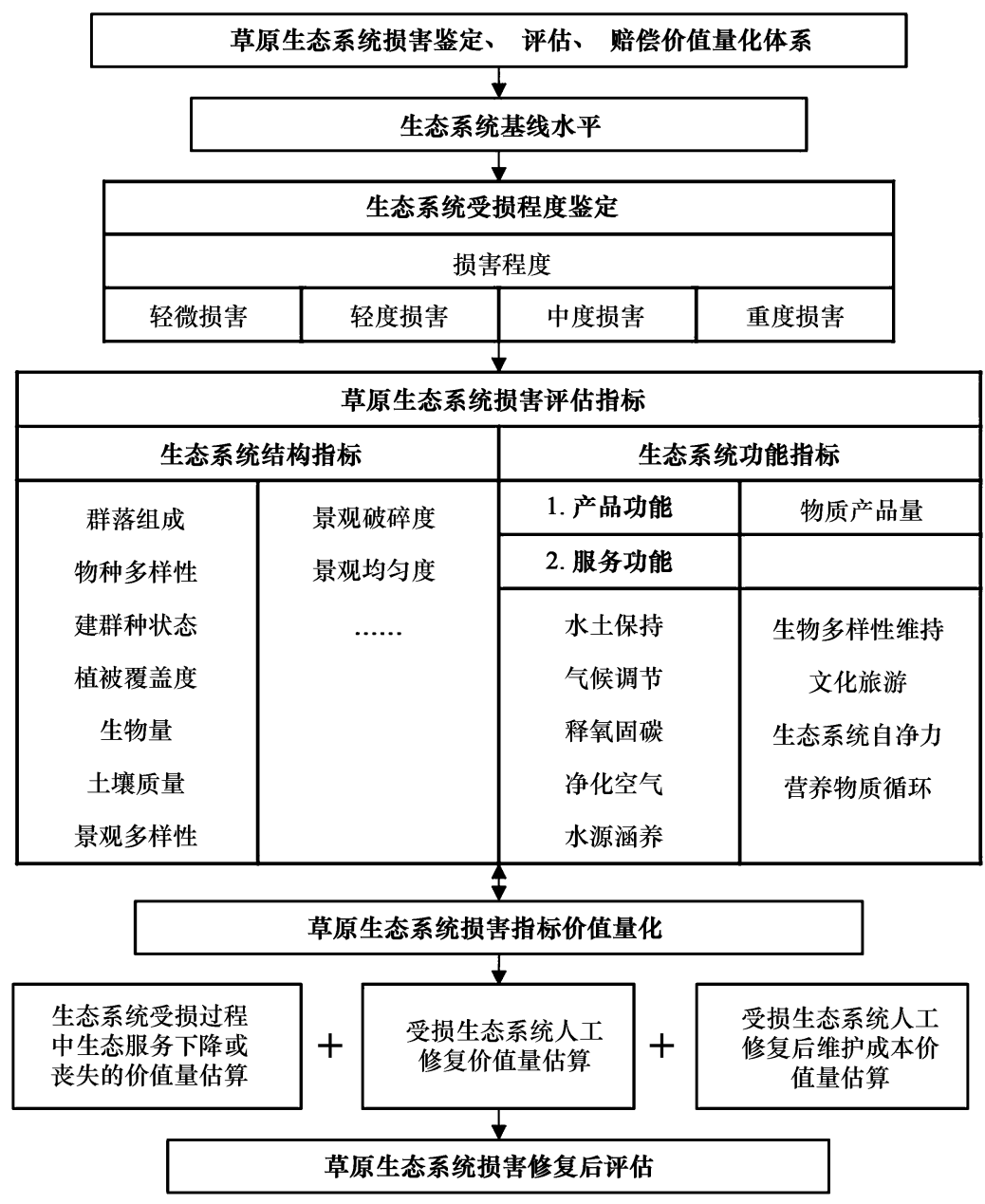

图 1 草原生态系统损害鉴定、评估、赔偿价值量化体系

Fig.1 The grassland ecosystem damage identification and assessment system 
量、土壤质量、景观多样性、景观均匀度及景观破碎度等; 功能指标包括产品功能和服务功能两个方面, 产品功 能主要包括生态系统为人类和社会直接或间接提供的产品,如:牧草、中药材、畜牧业产品、草下经济产品等; 服务功能主要包括草原生态系统的释氧固碳、气候调节、净化空气、水源涵养、水土保持、生物多样性维持、文 化旅游、生态系统自净能力、营养物质循环等 ${ }^{[26-29]}$ 。对于生态系统服务评价应根据损害行为的特点从物质量 或价值量两个角度分别进行考量 ${ }^{[30]}$ 。再次, 对受损生态系统各项指标进行价值量化, 进而判断其损害程度。

就目前司法实践来看, 对于生态系统损害赔偿主要以将受损生态系统恢复至基线水平或重建至人们理想 的水平所采取的各类人工修复成本为限,并未将从损害行为发生起至恢复到基线水平止或重建到人们满意水 平止生态产品和生态服务降低量的价值纳人赔偿范围, 同时也未将人工修复或重建工程后的维护成本费用纳 人到赔偿范围中 ${ }^{[31-32]}$ 。这种做法并未完整反映生态系统损害的全部价值, 因此在对损害进行价值量评估时, 还应将这两部分价值量纳人评估赔偿范围之中。

\section{3 草原生态系统损害赔偿价值量化的构成}

因生态系统损害活动是一个持续性过程,故损害赔偿价值量化应从损害活动发生后所造成的生态系统服 务下降或丧失的价值损失、受损生态系统人工修复价值、受损生态系统人工恢复价值及受损生态系统人工修 复后至形成稳定生态系统为止所需的维护费用三部分进行量化。

生态系统服务下降或丧失的价值损失可通过服务等值分析法进行量化,即通过建立生态破坏所导致生态 系统服务损失的折现量与恢复行为所恢复生态系统服务的折现量之间的等量关系来确定。

受损生态系统人工恢复价值可通过将生态系统恢复至极限水平需要开展的生态修复工程措施费用进行 计算, 同时还应包括损害行为开始至恢复基线水平的期间损害。

受损生态系统人工恢复价值及受损生态系统人工修复后至形成稳定生态系统为止所需的维护费用可通 过对修复后生态系统所需维护措施费用计算进行量化。

如果无法将受损生态系统恢复至基线水平或只能部分恢复,则可采用环境价值评估法对生态系统的永久 性损害进行价值评估。

\section{4 管理建议}

4.1 推动生态环境损害鉴定评估专门法律的制定

生态系统损害是生态环境损害的核心部分,生态环境损害鉴定评估工作作为一项司法实践活动,其性质 决定生态环境损害鉴定评估的各个环节必须依法进行。目前生态环境诉讼或非诉讼案件大多依靠《民法通 则》、《环境保护法》、《侵权责任法》、《民事诉讼法》及相关部门发布的指南等法律法规进行规制 ${ }^{[4]}$ 。目前我 国尚无一部针对生态环境损害的专门法律,更没有针对生态环境损害鉴定评估工作特点,用于全面规制生态 环境损害鉴定评估工作实施机制、技术方法、监督管理等内容的专门法律, 从而使生态环境损害鉴定评估工作 在具体操作时,没有上位法作为指导和支撑。尽管有关部门和各地已出台有关技术规范、管理规定等行政文 件,但无法为生态环境损害鉴定评估工作提供法律层面的保障。因此,制定一部具有导向性、规范性的专门法 律, 对于解决日益增长的生态环境诉讼或非诉讼案件极为必要。

制定《生态环境损害鉴定评估法》应就法律主体、鉴定评估范围、生态环境各领域所用技术方法、鉴定评 估程序、损害程度认定、责任承担、管理部门、鉴定评估机构资质、从业人员资格、法律援助、追责机制等内容加 以明确,并将程序法方面的内容纳人其中 ${ }^{[33-34]}$, 从而为生态环境损害鉴定评估工作的开展,提供全方位的法 律保障。

\section{2 完善生态环境损害鉴定评估技术体系}

我国生态环境损害鉴定评估技术研究始于 2011 年原环保部制定并发布《关于开展环境污染损害鉴定评 估工作的若干意见》, 随后中共中央办公厅、国务院、生态环境部相继发布《环境损害鉴定评估推荐方法(第 I 
版)》、《环境损害鉴定评估推荐方法(第 II 版)》、《生态环境损害赔偿制度改革试点方案》、《生态环境损害鉴 定评估技术指南总纲》、《生态环境损害鉴定评估技术指南损害调查》、《生态环境损害鉴定评估技术指南土壤 与地下水》及《生态环境损害鉴定评估技术指南地表水与沉积物 (征求意见稿)》。同时重庆、广东、江苏、江 西、内蒙古等省、自治区及直辖市也相继发布关于生态环境损害鉴定评估的相关实施方案或管理办法。这在 一定程度上为生态环境损害鉴定评估工作的开展及司法行政机关处理环境赔偿纠纷提供了指导。

但已有的生态环境损害鉴定评估技术体系仍存在一些不可忽视的问题,例如《技术指南总纲》对于鉴定 原则、工作方法规定较为笼统, 缺乏实际操作指导性; 《损害调查》就生态系统损害所需调查内容并未根据不 同生态系统类型进行分类规定, 可能导致确定损害程度不准确; 《分项技术指南》主要针对环境要素进行规 范, 目前尚未就生态系统损害鉴定评估发布专门技术指南,特别是在生态系统损害价值量评估方面过于局 限 ${ }^{[25]}$ 。因此, 还需立足国情, 将分散在各行业的技术规范整合到一起, 形成涵盖各环境要素及不同生态系统 类型的完整的生态环境损害鉴定评估技术体系。评估体系应针对不同生态系统设立不同的评估指标和评估 量, 同时应该建立具有可操作性的检测方法、指导意见及规范步骤。

4.3 完善损害评估量化标准及流程

由于生态系统损害扰动因素众多、影响范围和时间难以准确判定, 因此生态系统损害与传统意义上的环 境损害量化方式及流程存在较大差异, 应针对生态系统特点建立一套大尺度、多维度的损害量化方式,有些区 域数据全面, 宜选用多种方法相互验证, 最终确定损害程度, 有些区域难以到达或无法达到采集样点数据标 准, 只能根据参考点位的质量选择不同方法确定损害程度,而必要的确定顺序显得十分关键。目前主流的判 定方法包括统计点位法、模型法、专家判别法和历史数据法。但不同方法的适用范围有所差异,如果未受干扰 的区域占据大部分, 目标要求精确、人力财力充足, 区域空间尺度调查 (Spatial Network) 是最好的, 其次选择统 计点位法; 如果干扰区域占据大部分, 模型法或者回归法更适用; 如果其他方法不可用时, 历史数据法或专家 判别法则可以作为量化标准。

以草原生态系统为例, 我国草原区地广人稀, 历史监测数据普遍稀缺, 草原区种类繁多, 植被种类、土壤性 状差异巨大,因此在实际操作过程中根据不同草原的情况确定损害量化方式。内蒙古、青海、新疆草原地区面 积广大、人烟稀少、草原种类较为丰富, 但牧场退化面积大, 受自然条件影响较大, 宜采取统计点位法确定损害 程度;四川、湖南等南部地区草原受人类干扰较大,重金属含量较高,宜采取模型法推断草原的损害程度;西藏 地区草原由于气候恶劣, 地形复杂, 宜应用历史数据法、专家判别法等确定损害程度。采取哪种方法判定损害 程度需要结合当地生态条件、可用数据质量、生态损害程度共同决定, 可以选择一种方法确定, 也可以选择几 种不同方法组合相互验证来确定。

\section{5 结语}

生态系统损害鉴定评估是推进我国生态文明建设的重要保障,同时其作为生态环境损害司法实践中的重 要组成部分, 其特性决定损害鉴定评估工作的各个环节必须有法可依。健全生态系统损害等级划分及不同损 害等级的损害价值量评估, 对于生态环境损害鉴定评估工作具有重要的指导意义。我国目前尚无针对生态环 境损害鉴定评估专门立法, 对于生态环境损害鉴定评估工作的开展无法提供系统性法律支撑, 同时由于生态 环境损害鉴定评估技术体系的不完善,致使在实践过程中出现鉴定标准不统一、评估技术部完善、量化标准不 明确、鉴定意见遭质疑等多种问题。本文从受损生态系统能否恢复至生态系统基线水平或重建到人们理想的 生态系统水平角度出发, 分析草原生态系统损害状态及鉴定等级,并就草原生态系统鉴定评估指标及草原生 态系统损害评估指标价值的量化加以分析, 提出草原生态系统损害鉴定评估体系, 并从推动生态环境损害鉴 定评估法的制定和完善生态环境损害鉴定评估技术体系两个方面提出管理建议, 以期为生态环境损害鉴定评 估工作的完善提供科学参考,推进生态环境损害鉴定评估工作进一步发展。

总体而言, 草原生态系统损害鉴定、评估及赔偿价值量化体系的构建是面向草原生态系统损害评估管理 
的有益尝试,其对受损对象的鉴定, 及受损状况的评估, 以及受损赔偿的价值评估的把握有助于准确和全面的 反映草原生态系统损害的基本现状,也为损害的管理工作提供了理论基础和参考依据。然而,草原生态系统 损害评估工作是一个逐步细化的过程,尚需针对不同草原生态系统的类型之间评估指标的差异,进行因地制 宜的完善; 同时,草原生态系统损害鉴定评估体系, 不仅需要评估框架的建立, 针对损害基线的确认等细节工 作尚需进一步完善,对量化方法的选择也需要进一步优化,这都是未来研究需要完善的研究方向。

\section{参考文献( References) :}

[ 1 ] 吴钢, 曹飞飞, 张元勋, 张洪勋, 余志晟, 乔冰, 朱岩, 董仁才, 吴德胜, 高振会, 张逦嘉. 生态环境损害鉴定评估业务化技术研究. 生态 学报, 2016, 36(22): 7146-7151.

[ 2 ] 李嘉珣, 曹飞飞, 汪铭一, 吴钢. 参照点位法下的参照状态在草原生态系统损害基线判定中的应用分析. 生态学报, 2019, 39(19)： 6966-6973.

[ 3 ] Yan Y, Qian Y, Wang Z Y, Yang X Y, Wang H W. Ecological risk assessment from the viewpoint of surface water pollution in Xiamen City, China. International Journal of Sustainable Development \& World Ecology, 2018, 25(5) : 403-410.

[ 4 ] 於方, 张衍壆, 徐伟攀.《生态环境损害鉴定评估技术指南总纲》解读. 环境保护, 2016, 44(20): 9-11.

[ 5 ] 环境保护部. 生态环境损害鉴定评估技术指南总纲. 环境保护部, 2016. http://www. mee. gov. cn/gkml/hbb/bgt/201607/t20160705_ 357139.html

[ 6 ] 生态环境部. 生态环境损害鉴定评估技术指南土壤与地下水. 生态环境部, 2018. http://www.mee.gov.cn/xxgk2018/xxgk/xxgk05/201812/ t20181225_685946.html

[ 7 ] Costanza R, d'Arge R, de Groot R, Farber S, Grasso M, Hannon B, Limburg K, Naeem S, O'Neill R V, Paruelo J, Raskin R G, Sutton P, van den Belt M. The value of the world's ecosystem services and natural capital. Nature, 1997, 387(6630): 253-260.

[ 8 ] Cairns J Jr, Niederlehner B R. Ecosystem health concepts as a management tool. Journal of Aquatic Ecosystem Health, 1995 , 4(2) : 91-95.

[ 9 ] 欧阳志云, 王如松, 赵景柱. 生态系统服务功能及其生态经济价值评价. 应用生态学报, 1999, 10(5): 635-640.

[10］於方, 赵丹, 王膑, 张志宏.《生态环境损害鉴定评估技术指南 土壤与地下水》解读. 环境保护, 2019, 47(5): 19-23.

[11] 张红振, 曹东, 於方, 王金南, 齐雯, 贾倩, 张天柱, 骆永明. 环境损害评估: 国际制度及对中国的启示. 环境科学, 2013, 34(5)： 1653- 1666 .

[12] 唐小晴, 张天柱. 环境损害赔偿之关键前提: 因果关系判定. 中国人口・资源与环境, 2012, 22(8): 172-176.

[13] Bishop R C, Boyle K J, Carson R T, Chapman D, Hanemann W M, Kanninen B, Kopp R J, Krosnick J A, List J, Meade N, Paterson R, Presser S, Smith V K, Tourangeau R, Welsh M, Wooldridge J M, DeBell M, Donovan C, Konopka M, Scherer N. Putting a value on injuries to natural assets: the BP oil spill. Science, 2017, 356(6335): 253-254.

[14] Desvousges W H, Gard N, Michael H J, Chance A D. Habitat and resource equivalency analysis: a critical assessment. Ecological Economics, 2018, 143 : 74-89.

[15] Zafonte M, Hampton S. Exploring welfare implications of resource equivalency analysis in natural resource damage assessments. Ecological Economics, 2007, 61(1): 134-145.

[16] 包芯, 李涛, 张欣怡, 付晓, 赵宇, 唐明方, 邓红兵. 森林生态系统损害评估体系与管理制度研究. 生态学报, 2021, 41(3): 924-933.

[17] 于恩逸, 崔宁, 吴迪, 唐明方, 付晓, 吴钢. 草原生态环境损害因果关系判定路径. 生态学报, 2021, 41(3) : 943-948.

[18] 崔宁, 于恩逸, 李爽, 唐明方, 吴钢. 基于生态系统敏感性与生态功能重要性的高原湖泊分区保护研究一一达里湖流域为例. 生态学 报, 2021, 41(3): 949-958.

[19] Attiwill P M. The disturbance of forest ecosystems: the ecological basis for conservative management. Forest Ecology and Management, 1994, 63 $(2 / 3): 247-300$.

[20] Barnthouse L W, Stahl Jr R G. Quantifying natural resource injuries and ecological service reductions: challenges and opportunities. Environmental Management, 2002, 30(1): 1-12.

[21］侯向阳, 李西良, 高新否. 中国草原管理的发展过程与趋势. 中国农业资源与区划, 2019, 40(7): 1-10.

[22］赵同谦, 欧阳志云, 贾良清, 郑华. 中国草地生态系统服务功能间接价值评价. 生态学报, 2004, 34(6): 1101-1110.

[23] 谢高地, 张钇锂, 鲁春霞, 郑度, 成升鬼. 中国自然草地生态系统服务价值. 自然资源学报, 2001, 16(1): 47-53.

[24] 卫草源. 草原生态环境损害赔偿制度研究. 中国畜牧业, 2016, (24) : 70-71.

[25]马克明, 孔红梅, 关文涁, 傅伯杰. 生态系统健康评价: 方法与方向. 生态学报, 2001, 21 (12): 2106-2116.

[26] 尹剑慧, 卢欣石. 草原生态服务价值核算体系构建研究. 草地学报, 2009, 17(2): 174- 180.

[27]叶金金, 周华坤, 赵新全, 温军, 陈哲, 段吉闯. 草地生态系统健康研究述评. 草业科学, 2011, 28(4): 549-560.

[28］于遵波. 草地生态系统价值评估及其动态模拟 [D]. 北京: 中国农业大学, 2005.

[29] 闵庆文, 刘寿东, 杨霞. 内蒙古典型草原生态系统服务功能价值评估研究. 草地学报, 2004, 12(3)：165-169，175-175.

[30] 赵景柱, 肖寒, 吴刚. 生态系统服务的物质量与价值量评价方法的比较分析. 应用生态学报, 2000, 11(2): 290-292.

[31] 於方, 张红振, 牛坤玉, 谢光轩. 我国的环境损害评估范围界定与评估方法. 环境保护, 2012, (5): 25- 29.

[32］鲁俊华. 公益诉讼中环境损害赔偿数额确定之反思. 中国检察官, 2019, (12) : 70-74.

[33］孙来晶. 环境损害司法鉴定的发展与困境. 法制与社会, 2019, (17)：100-101.

[34] 於方, 齐雯, 张志宏. 《生态环境损害鉴定评估技术指南损害调查》解读. 环境保护, 2016, 44(24) : 16-19. 